\title{
Long-range spin jump diffusion revealed by dynamic light scattering
}

\author{
S. Cronenberger, ${ }^{1}$ H. Boukari $\odot,{ }^{2}$ D. Ferrand, ${ }^{2}$ J. Cibert $\odot,{ }^{2}$ and D. Scalbert $\oplus^{1}$ \\ ${ }^{1}$ Laboratoire Charles Coulomb, UMR 5221 CNRS/Université de Montpellier, F-34095 Montpellier, France \\ ${ }^{2}$ Université Grenoble Alpes, CNRS and Grenoble INP, Institut Néel, F-38000 Grenoble, France
}

(Received 11 December 2020; revised 17 May 2021; accepted 18 May 2021; published 28 May 2021)

\begin{abstract}
Spatiotemporal spin noise spectroscopy is combined with dynamic light scattering in order to reach spatial resolutions down to $\sim \lambda / 10$. Applied to a system of localized electron spins, an insulating n-doped CdTe layer, this allows us to reveal long spin jump distances $\ell \sim 2.7 \mu \mathrm{m}$. Spin noise spectra at large wave vectors $q(q \ell \gg 1)$ provide a snapshot of the spin dynamics before jump (therefore not affected by spin motion), while at smaller $q$, spin motion sets in. This allows us to unravel the contributions of spin-orbit and hyperfine fields in the electron spin relaxation and to determine self-consistently all parameters relevant to the spin dynamics. We propose a phenomenological equation inspired by studies of atomic jump diffusion by neutron scattering, which includes the relevant spin relaxation mechanisms and the effect of time of flight of the spin fluctuation across the laser spot. This modeling reproduces all experimental results.
\end{abstract}

DOI: 10.1103/PhysRevB.103.205208

\section{INTRODUCTION}

Donor electron spins in semiconductors are being actively studied as they hold great promise in the context of quantum technologies [1-3]. Such spins are among the most coherent quantum systems in the solid state and can be efficiently exploited by using advanced semiconductor technology $[4,5]$. Long-distance displacement of individual electron spins is another key ingredient for scalability in a spin-based semiconductor quantum circuit. This is one option to perform long-range interaction between distant spin qubits [6,7]. It has been proposed to move the electron spin coherently over large distances in a well-defined tunnel coupled array of empty donors or dots. In practice, this has been realized in quantum-dot arrays containing up to four electrostatic dots in GaAs/GaAlAs heterostructures, with size not exceeding a few micrometers [8-10].

In this paper, we demonstrate spontaneous spin jumps between donors separated by a few micrometers in a many-body interacting disordered spin system formed by isolated donors. Although the jumps are, in this situation, uncontrolled, understanding the underlying mechanisms will open new prospects for coherent long-distance displacement of spins in semiconductors and help to determine to what extent unwanted jumps can be suppressed.

Experimentally, we reveal the jump-diffusion regime by passively probing the stochastic spin fluctuations at thermal equilibrium with off-resonant light. This noise-based approach, issuing from atomic physics [11-13] and quantum optics [14-16], has been also actively developed in semiconductors since $2005[17,18]$. In these materials, homodyne and heterodyne detection opened the route to nearly perturbationfree measurements [19-21]. Different theoretical proposals have pointed out the interest in probing spatial spin correlations both in semiconductors [22-24] and in cold fermionic gases [25]. Recently, we experimentally realized spatiotemporal spin noise spectroscopy applied to semiconductors (ST-SNS) and obtained the wave-vector-resolved dynamical spin response function of electrons [26,27]. Here, we further extend the accessible range of wave vectors $q$ in the spirit of dynamic light scattering. This allows us to go beyond the $q$ values which have been explored so far [28-33]. This way, we clearly reveal a deviation from the classical $q^{2}$ diffusion law and observe a plateau at large $q$; these findings are the signature of the jump-diffusion regime.

Jump diffusion is a stochastic process that involves both discrete jumps and continuous motion. It finds applications in many different fields ranging from physics $[34,35]$ to epidemiology [36], biology [37,38], and risk management in financial markets [39,40]. In condensed matter physics, jump-diffusion processes control, e.g., the atomic diffusion in crystals and in some liquids, such as water and lead $[41,42]$. Because jump diffusion normally occurs at the atomic scale, it has been extensively studied by means of quasielastic neutron scattering $[43,44]$. We rely on this large body of work to develop below a model which gives the first complete description of spatiotemporal spin dynamics in an ensemble of disordered localized spins.

Among the presently investigated materials it has been suggested that wide-gap II-VI semiconductors combine most of the advantages of solid-state systems [45-47]: high natural abundance of isotopes with zero nuclear spins, which also permits isotopic purification and reduction of decoherence of electron spins due to hyperfine interaction [48]; a direct band gap allowing for an efficient spin-photon entanglement; and large binding energies which confer high thermal stability to donor-bound electrons. Long spin coherence $T_{2}$ times up to $50 \mu \mathrm{s}$, a prerequisite for quantum computing, have been measured, for example, in $\mathrm{ZnO}$ [47] and could be further extended with isotope purification. In fluorine-doped $\mathrm{ZnSe}$, 
inhomogeneous spin dephasing times up to 33 ns were observed below $40 \mathrm{~K}$ [45].

We demonstrate jump diffusion in an insulating n-CdTe sample by resolving effective spin jumps over distances $\ell \sim$ $2.7 \mu \mathrm{m}$. ST-SNS alone is not sufficient for this task because high spatial frequencies are cut, due to the finite resolution of the imaging system [26]. This hurdle is bypassed if the dynamically scattered light is collected in an arbitrary direction. Here, we combine three detection schemes: classical SNS ( $q=0$ in forward scattering), ST-SNS $\left(q \leqslant 3 \mu \mathrm{m}^{-1}\right)$, and backscattering SNS $\left(q_{b}=4 \pi n / \lambda \simeq 50 \mu \mathrm{m}^{-1}\right.$, with $n$ being the refractive index and $\lambda$ being the wavelength). High spatial frequencies up to $q_{b}$ become accessible, corresponding to a spatial resolution $\sim \lambda / 10$.

In the next section we present our model for the wavevector-resolved spin noise spectra. There, we derive a phenomenological equation, which incorporates the structure factor necessary to account for the light scattering in an arbitrary direction, and the different spin decoherence mechanisms. Section III is devoted to the experimental results and their analysis with the model developed in Sec. II. Finally, we conclude in Sec. IV.

\section{WAVE-VECTOR-RESOLVED SPIN NOISE SPECTRA IN THE RANDOM-WALK APPROACH}

Our calculation is inspired by previous theories [41,42,4952]. In the same spirit, we compute the spin polarization decay within a random-walk approach and express it as an infinite sum over the number of spin jumps between localized sites. Our approach, unlike theories aimed at calculating the spin resonance or spin noise line shapes, introduces the optical phase factor due to light scattering at a finite angle.

\section{A. Phase coherence decay}

The decay of phase coherence of electron spin rotation is calculated in the presence of jump diffusion taking into account the jump length distribution.

\section{The random-walk description}

We first recall the main lines of the random-walk model for the decay of phase coherence of rotating spins which perform jumps in an ideal crystal lattice [50]. The spins precess with the Larmor frequency $\omega_{0}$ under the influence of an external magnetic field. The phase coherence of the rotation of different spins is gradually diminished through the random orientation of nuclear fields at different sites. In the presence of spin jump diffusion, the individual spins sense more or less averaged nuclear fields, depending on the jump rate. The decay of the polarization is reduced compared with the case of no diffusion; that is, motional narrowing occurs. The basic feature of the model is a separation of the polarization decay $P(t)$ into the contribution of a fixed number of generalized jumps,

$$
P(t)=\sum_{l=0}^{\infty} R_{l}(t)
$$

$R_{l}(t)$ is the contribution to the polarization decay of all spins which have made $l$ jumps until the time $t$. The initial condition is $P(t=0)=1$. In particular, we have

$$
R_{0}(t)=P_{0}(t) \exp \left(-t / \tau_{c}\right)
$$

where $\tau_{c}$ is the residence time at each site and $\exp \left(-t / \tau_{c}\right)$ is the probability that the spin has performed no jump until t. $P_{0}(t)=\exp \left(-\frac{1}{2} \sigma^{2} t^{2}\right)$ is the decay of the phase coherence due to the Gaussian distribution ( $\sigma^{2}$ second moment) of spin precession frequencies at different localized sites and given by the ensemble average over many spins. If we assume no correlations between the precession frequencies at different sites, the ensemble average can be performed at each jump, and the decay of phase coherence at each step can be calculated as a function of $R_{0}(t)$; we have, after one jump,

$$
R_{1}(t)=\int_{0}^{t} d t^{\prime} R_{0}\left(t-t^{\prime}\right) \frac{1}{\tau_{c}} R_{0}\left(t^{\prime}\right),
$$

and after $l$ jumps

$$
R_{l}(t)=\int_{0}^{t} d t^{\prime} R_{0}\left(t-t^{\prime}\right) \frac{1}{\tau_{c}} R_{l-1}\left(t^{\prime}\right),
$$

and the series in Eq. (1) is summed up by the integral equation

$$
P(t)=R_{0}(t)+\frac{1}{\tau_{c}} \int_{0}^{t} d t^{\prime} R_{0}\left(t-t^{\prime}\right) P\left(t^{\prime}\right) .
$$

The integral equation can be solved by Laplace transformation

$$
\tilde{P}(s)=\tilde{R}_{0}(s) \sum_{l=0}^{\infty}\left(\frac{1}{\tau_{c}} \tilde{R}_{0}(s)\right)^{l}=\frac{\tilde{R}_{0}(s)}{1-\frac{1}{\tau_{c}} \tilde{R}_{0}(s)},
$$

where $\tilde{P}(s)$ and $\tilde{R}_{0}(s)$ are given by

$$
\begin{gathered}
\tilde{P}(s)=\int_{0}^{\infty} d t \exp (-s t) P(t), \\
\tilde{R}_{0}(s)=\int_{0}^{\infty} d t \exp (-s t) R_{0}(t) \\
=\frac{\sqrt{\pi}}{\sqrt{2} \sigma} \exp \left[\frac{\left(s+\frac{1}{\tau_{c}}\right)^{2}}{2 \sigma^{2}}\right] \operatorname{erfc}\left(\frac{s+\frac{1}{\tau_{c}}}{\sqrt{2} \sigma}\right) .
\end{gathered}
$$

$\tilde{R}_{0}(s)$ can be conveniently expressed with the Faddeeva function, for which efficient implementations exist for numerical calculations,

$$
\mathcal{W}(z)=e^{-z^{2}} \operatorname{erfc}(-i z)
$$

so that

$$
\tilde{R}_{0}(s)=\frac{\sqrt{\pi}}{\sqrt{2} \sigma} \mathcal{W}\left(\frac{i\left(s+\frac{1}{\tau_{c}}\right)}{\sqrt{2} \sigma}\right)
$$

$\tilde{P}(s)$ is related to the spin noise spectrum $S(\omega)$,

$$
S(\omega)=\frac{1}{\pi} \operatorname{Re}\left\{\tilde{P}\left[s=i\left(\omega-\omega_{0}\right)\right]\right\} .
$$

An angular rotation $R_{\mathrm{so}}$ occurs from the jump event when spin-orbit coupling is present. In the case where all angular 
rotations due to spin orbit are the same at each spin jump, Eq. (6) becomes [51]

$$
\begin{gathered}
\tilde{P}(s)=\tilde{R}_{0}(s) \sum_{l=0}^{\infty} R_{\mathrm{so}}^{l}\left(\frac{1}{\tau_{c}} \tilde{R}_{0}(s)\right)^{l} \\
=\frac{\tilde{R}_{0}(s)}{1-R_{\mathrm{so}} \frac{1}{\tau_{c}} \tilde{R}_{0}(s)},
\end{gathered}
$$

where $R_{\mathrm{so}}=1-\frac{4}{3} \sin ^{2}\left(\frac{\gamma}{2}\right)$ for the spatially averaged spinorbit rotation.

One can incorporate an intrasite relaxation independent of hopping by multiplying the right-hand side of Eq. (2) by $\exp \left(-v_{s} t\right)$, with $v_{s}$ being the intrasite spin relaxation rate. Thus, with an intrasite relaxation, $\tilde{R}_{0}(s)$ becomes $\tilde{R}_{0}\left(s+v_{s}\right)$.

\section{Optical phase factor}

In the following we will introduce the effect of a spatial phase factor which appears when the spin system is probed at scattered angles, i.e., when the signal is detected in a direction different from that of the incident light. This kind of configuration of noise measurement is usually called dynamic light scattering (DLS) measurement. The spatial phase factor comes from "interferences" between the incident light and the scattered light; it is then equal to the relative optical phase between the incident and scattered fields. A particle at a given position $\mathbf{r}_{\mathbf{i}}$ scatters the incident plane wave, initially propagating in the direction $\mathbf{u}_{\mathbf{0}}$, in all directions. When detected far from the sample at a position $\mathbf{r}_{\mathbf{d}}$, the scattered light has an additional phase factor $e^{-i \mathbf{q} \cdot \mathbf{r}_{\mathbf{i}}}$, which is equal to 1 when detected in the direction of the incident light, where $\mathbf{q}=\mathbf{q}_{\mathbf{0}}-\mathbf{q}_{\mathbf{d}}$, with $\mathbf{q}_{\mathbf{0}}$ and $\mathbf{q}_{\mathbf{d}}$ being the incident and scattered wave vectors, respectively. $q=\frac{4 \pi}{\lambda} \sin \left(\frac{\theta}{2}\right)$, with $\theta=\operatorname{acos}\left(\frac{\mathbf{u}_{0} \cdot\left(\mathbf{r}_{\mathbf{d}}-\mathbf{r}_{\mathbf{i}}\right)}{\left|\mathbf{u}_{0}\right|\left|\left(\mathbf{r}_{\mathbf{d}}-\mathbf{r}_{\mathbf{i}}\right)\right|}\right)$. Thus, when a particle at a given time $t$ and at a given position $r_{i}$ jumps to another position at another time, its phase changes; then the phase coherence diminishes at each jump. This results in an additional broadening of the line described by Eqs. (6) or (13), which contains the spatial jump structure.

The Kehr-Richter-Honig model described in Sec. II A 1 has been developed assuming a uniform lattice, but it is valid (except for the inclusion of the spin-orbit coupling) for jumps with different lengths while the system is probed in transmission. We revisit the steps of the random-walk description leading to Eq. (6) taking into account the spatial distribution of jumps and the optical spatial phase factor appearing in a DLS measurement. Let us consider that the jumps occur between a given site and $N$ others. A spin at a position $\mathbf{r}_{\mathbf{i}}$ can jump to $N$ other positions $\mathbf{r}_{\mathbf{i}}+\mathbf{d}_{v}$, where $\mathbf{d}_{v}(v=1,2, \ldots, N)$ is a set of $N$ jump vectors connecting the site with the others. The jump rate from a site $\mathbf{r}_{\mathbf{i}}$ to a site $\mathbf{r}_{\mathbf{i}}+\mathbf{d}_{v}$ is $\Gamma_{\nu}$. We assume that the spatial structure of the jumps is the same whatever the site position, i.e., a spin initially at $\mathbf{r}_{\mathbf{i}}$ after one jump will occupy $N$ different sites at $\mathbf{r}_{\mathbf{i}}+\mathbf{d}_{v}$ depending on the jump rates and after a second jump it will occupy $\sim N^{2} / 2$ sites at positions $\mathbf{r}_{\mathbf{i}}+\mathbf{d}_{v}+\mathbf{d}_{v^{\prime}}\left(v, v^{\prime}=1,2, \ldots, N\right)$ (the factor $1 / 2$ accounts for the fact that the same site can be reached by two different paths). At a site a spin spends a time $\tau_{c}$ with $1 / \tau_{c}=\sum_{v=1}^{N} \Gamma_{v}$, and the time required to jump from site to site is neglected. For zero jump, Eq. (2) remains unchanged, but after one jump we have to take into account the phase variation $\delta \phi=\mathbf{q} \cdot \mathbf{d}_{v}$ due to the spatial displacement $\mathbf{d}_{v}$ of the spin; then Eq. (3) becomes

$$
\begin{aligned}
& R_{1}(t)=\int_{0}^{t} d t^{\prime} R_{0}\left(t-t^{\prime}\right) \sum_{\nu=1}^{N} \Gamma_{\nu} e^{-i \mathbf{q} \cdot \mathbf{d}_{v}} R_{0}\left(t^{\prime}\right) \\
& =\tau_{c} \sum_{\nu=1}^{N} \Gamma_{\nu} e^{-i \mathbf{q} \cdot \mathbf{d}_{\nu}} \int_{0}^{t} d t^{\prime} R_{0}\left(t-t^{\prime}\right) \frac{1}{\tau_{c}} R_{0}\left(t^{\prime}\right)
\end{aligned}
$$

and the Laplace transform of the series in Eq. (1) is written as

$$
\begin{gathered}
\tilde{P}(s)=\tilde{R}_{0}(s) \sum_{l=0}^{\infty}\left(\tau_{c} \sum_{\substack{\nu=1 \\
\tilde{R}_{0}(s)}}^{N} \Gamma_{\nu} e^{-i \mathbf{q} \cdot \mathbf{d}_{\nu}} \frac{1}{\tau_{c}} \tilde{R}_{0}(s)\right)^{l} \\
=\frac{1-\left[1-\tau_{c} \Gamma(\mathbf{q})\right] \frac{1}{\tau_{c}} \tilde{R}_{0}(s)}{1-}
\end{gathered}
$$

with $\tilde{R}_{0}(s)$ given by Eq. (8) and $\Gamma(\mathbf{q})$ being the Fourier transform of the transition rate matrix $\Gamma_{i, i^{\prime}}, \Gamma(q)=$ $\sum_{i, i^{\prime}} \Gamma_{i, i^{\prime}} e^{-i \mathbf{q} \cdot\left(\mathbf{r}_{\mathbf{i}}-\mathbf{r}_{\mathbf{i}^{\prime}}\right)}=\sum_{v=1}^{N} \Gamma_{v}\left(1-e^{-i \mathbf{q} \cdot \mathbf{d}_{v}}\right) . \Gamma_{i, i^{\prime}}$ satisfy the master equation of the conditional probability $P\left(\mathbf{r}_{\mathbf{i}} t \mid \mathbf{0 0}\right)$ of finding a particle at site $\mathbf{r}_{\mathbf{i}}$ at time $t$ when it was at site $\mathbf{0}$ at time 0 ,

$$
\frac{d}{d t} P\left(\mathbf{r}_{\mathbf{i}} t \mid \mathbf{0 0}\right)=\sum_{i^{\prime}} \Gamma_{i, i^{\prime}} P\left(\mathbf{r}_{\mathbf{i}^{\prime}} t \mid \mathbf{0 0}\right)
$$

with

$$
\Gamma_{i, i^{\prime}}= \begin{cases}\sum_{v} \Gamma_{v} & \text { if } \mathbf{r}_{\mathbf{i}}=\mathbf{r}_{\mathbf{i}^{\prime}} \\ -\Gamma_{v} & \text { if } \mathbf{r}_{\mathbf{i}}-\mathbf{r}_{\mathbf{i}^{\prime}}=\mathbf{d}_{v} \\ 0 & \text { otherwise. }\end{cases}
$$

The master equation has a convolution form which takes a more practical form in Fourier space,

$$
\frac{d}{d t} P(\mathbf{q}, t)=-\Gamma(\mathbf{q}) P(\mathbf{q}, t),
$$

where $P(\mathbf{q}, t)=\sum_{i} e^{i \mathbf{q} \cdot \mathbf{r}_{\mathbf{i}}} P\left(\mathbf{r}_{\mathbf{i}} t \mid \mathbf{0 0}\right)=e^{-\Gamma(\mathbf{q}) t}$.

The form of $\Gamma(\mathbf{q})$ has been calculated for various situations such as atom or molecule diffusion in solids or in liquids. One can name the well-known models of ChudleyElliott (CE) [41], Singwi-Sjölander (SS) [42], and Hall-Ross (HR) [53], largely used in neutron scattering theories and experiments in liquids. These models are called jump-diffusion models where the particle is in its equilibrium position for a mean time $\tau_{c}$ and then diffuses by continuous motion for a mean time $\tau_{d}$. When $\tau_{c} \gg \tau_{d}$, the diffusion takes place as successive jumps. The different models have been developed for different jump length distributions. The CE model assumes jumps of fixed distance but in random directions. In the SS model the particle has a probability $e^{-t / \tau_{d}}$ to remain in diffusive motion; this leads to an exponential jump length distribution as given in Fig. 1 [54]. The HR model deals with a Gaussian jump length distribution. In Fig. 1 the forms of $\Gamma(q)$ and of the spatial jump distribution $\rho(r)$ are given for the different models. One can note that, whatever the model, in the small- $q$ limit we recover the $q^{2}$ law for continuous diffusion for $\Gamma(q)$ and in the large- $q$ limit all models yield $\Gamma(q)=\frac{1}{\tau_{c}}$. 


\begin{tabular}{|c|c|c|c|}
\hline MODELS & $\rho(r)$ & $\Gamma(q)$ & $\overline{l^{2}}$ \\
\hline $\begin{array}{l}\text { Hypercubic } \\
\text { Lattices } \\
\text { Discrete jumps }\end{array}$ & & $\begin{array}{l}\frac{1}{\tau_{c}}\left[1-\frac{2}{n_{n}} \sum_{j=1}^{d} \cos \left(q_{j} l_{0}\right)\right] \\
n_{n} \text { : nearest neighborsnumber } \\
d: \text { dimensionality }\end{array}$ & $l_{0}^{2}$ \\
\hline $\begin{array}{l}\text { CE Model : } \\
\text { Fixed jump length } \\
\text { in random directions }\end{array}$ & $\delta\left(r-l_{0}\right)$ & $\frac{1}{\tau_{c}}\left(1-\frac{\sin \left(q l_{0}\right)}{q l_{0}}\right)$ & $l_{0}^{2}$ \\
\hline $\begin{array}{l}\text { HR Model : } \\
\text { Gaussian distribution } \\
\text { of jump lengths }\end{array}$ & $\sqrt{\frac{2}{\pi}} \frac{r^{2} e^{-r^{2} / 2 l_{0}^{2}}}{l_{0}^{3}}$ & $\frac{1}{\tau_{c}}\left(1-e^{-q^{2} l_{0}^{2} / 2}\right)$ & $3 l_{0}^{2}$ \\
\hline $\begin{array}{l}\text { SS Model: } \\
\text { Lorentzian distribution } \\
\text { of jump lengths }\end{array}$ & $\frac{r e^{-r / l_{0}}}{l_{0}^{2}}$ & $\frac{1}{\tau_{c}} \frac{q^{2} l_{0}^{2}}{1+q^{2} l_{0}^{2}}$ & $6 l_{0}^{2}$ \\
\hline
\end{tabular}

FIG. 1. The Fourier transform of the transition rate matrix $\Gamma(q)$ for a hypercubic arrangement of sites (chain, square lattice, simple cubic lattice, etc.) and for different models of jump diffusion with a distribution of jump lengths $\rho(r) . \overline{l^{2}}$ is the mean-square displacement, which is related to the characteristic jump length $l_{0}$ usually used in the models.

\section{B. Interplay of hyperfine interaction and spatially resolved spin jump distribution}

\section{Motional narrowing suppression: Probing no jump}

The interplay of the hyperfine interaction and carrier hopping in spin dynamics was studied in works on muon diffusion [50] (approach used in this paper) and in molecular systems [51,55,56]. More recently, an analytical expression of the spin noise spectrum has been derived for an ensemble of localized electrons, taking into account nuclear effects and electron hopping between the localized sites [52]. We have not seen reports of the interplay of the hyperfine interaction and spin jump in a configuration of DLS measurement. We will discuss some behaviors suggested by the wavevector-dependent spin noise spectrum $S_{0}(\omega, \mathbf{q})$ deduced from Eqs. (18) and (12),

$$
S_{0}(\omega, \mathbf{q})=\frac{1}{\pi} \operatorname{Re}\left\{\frac{\tilde{R}_{0}(s)}{1-\left[1-\tau_{c} \Gamma(\mathbf{q})\right] \frac{1}{\tau_{c}} \tilde{R}_{0}(s)}\right\},
$$

with $s=i\left(\omega-\omega_{0}\right)$.

First, in a transmission measurement with $q=0, \Gamma(0)=0$, and Eq. (21) reduces to the real part of Eq. (6), which reproduces the Gaussian decay of the polarization for large $\sigma \tau_{c}$ due to hyperfine interaction and motional narrowing inducing exponential decay for small $\sigma \tau_{c}$ [50]. For signals detected in a direction different from the incident light, for intermediate $\sigma \tau_{c}$, and for not-too-large wave vectors, the spin noise line broadens in a peculiar way. As known in motional narrowing, when $\sigma \tau_{c}$ decreases, the line shape changes from Gaussian to Lorentzian in such a way that the Gaussian part decreases and the Lorentzian part increases. At intermediate $\sigma \tau_{c}$, when the line shape is partly Gaussian and partly Lorentzian, an increase in $q$ broadens the spin noise line with an increase in the Gaussian part rather than the Lorentzian part. This kind of behavior is usually encountered with an increase in $\sigma \tau_{c}$ because the spins become more and more localized. The fact that we observe a similar behavior for the line shape when $q$ increases suggests that we look at spins which experience some but not all jumps of the distribution. The detection selects some diffusion paths; thus the spins seem more localized. Moreover, when $q$ has a value $q_{c}$ such that $\Gamma\left(q_{c}\right)=1 / \tau_{c}$, Eq. (21) reduces to

$$
S_{0}\left(\omega, \mathbf{q}_{\mathbf{c}}\right)=\frac{1}{\pi} \operatorname{Re}\left\{\tilde{R}_{0}(s)\right\} .
$$

Then for large wave vectors, in the case of continuous jump length distributions or for periodic values of $q$ in the case of jump on uniform lattices, the spin motion is suppressed by the detection configuration, i.e., the spatial optical phase averages out the spin jump distribution, and the spin dynamics before the first jump is measured. Thus no motional narrowing occurs when $\sigma \tau_{c}$ decreases, and the Gaussian decay of the polarization is accelerated by the probability that the spin leaves its equilibrium position.

\section{Spin-orbit coupling}

The inclusion of angular rotation due to spin-orbit coupling is quite simple if the diffusive motion is a direct hopping between nearest neighbors on uniform lattices because the hopping rotation is independent of the time (it appears only when the hop occurs) and it is independent of space since the rotation is the same at each hop. Thus the inclusion of a polarization decay due to spin-orbit coupling $R_{\text {so }}$ in Eq. (15) is obvious, and we get

$$
\begin{aligned}
R_{1}(t) & =R_{\text {so }} \int_{0}^{t} d t^{\prime} R_{0}\left(t-t^{\prime}\right) \sum_{\nu=1}^{N} \Gamma_{\nu} e^{-i \mathbf{q} \cdot \mathbf{d}_{v}} R_{0}\left(t^{\prime}\right) \\
& =R_{\mathrm{So}} \tau_{c} \sum_{\nu=1}^{N} \Gamma_{\nu} e^{-i \mathbf{q} \cdot \mathbf{d}_{\nu}} \int_{0}^{t} d t^{\prime} R_{0}\left(t-t^{\prime}\right) \frac{1}{\tau_{c}} R_{0}\left(t^{\prime}\right)
\end{aligned}
$$

the Laplace transform of the series in Eq. (1) is written as

$$
\tilde{P}(s)=\frac{\tilde{R}_{0}(s)}{1-R_{\mathrm{so}}\left(1-\tau_{c} \Gamma^{(u l)}(\mathbf{q})\right) \frac{1}{\tau_{c}} \tilde{R}_{0}(s)},
$$

with $\Gamma^{(u l)}(\mathbf{q})$ being the diffusion broadening for uniform lattices, $\Gamma^{(u l)}(\mathbf{q})=\frac{1}{\tau_{c}}\left\{1-\frac{1}{3}\left[\cos \left(q_{x} a\right)+\cos \left(q_{y} a\right)+\cos \left(q_{z} a\right)\right]\right\}$, for a simple cubic lattice of spacing $a$. It is interesting to note that if $\Gamma^{(u l)}(\mathbf{q})=1 / \tau_{c}$, then $\tilde{P}(s)$ reduces to $\tau_{c} \tilde{R}_{0}(s)$, which corresponds to the situation explained in the previous section. The spin-orbit rotation is suppressed because the correlations are lost (by the spatial averaging by the optical spatial phase) before the spin reaches its second equilibrium position (its equilibrium position after one jump). One can expect similar behavior at large $q$ in the case of continuous jump length distributions, i.e., spin-orbit-induced broadening vanishes when $\Gamma(q)=1 / \tau_{c}$. Therefore the hopping-induced rotation will be space dependent leading to a wave-vector dependency, which would be convoluted with $1-\tau_{c} \Gamma(\mathbf{q})$ as suggested by the term $\sum_{\nu=1}^{N} R_{\mathrm{so}, \nu} \Gamma_{\nu} e^{-i \mathbf{q} \cdot \mathbf{d}_{v}}$ in the equation below taking into 
account the jump length dependency of $R_{\mathrm{so}}$ in the expression of $R_{1}(t)$,

$$
\begin{aligned}
R_{1}(t) & =\int_{0}^{t} d t^{\prime} R_{0}\left(t-t^{\prime}\right) \sum_{\nu=1}^{N} R_{\mathrm{so}, \nu} \Gamma_{\nu} e^{-i \mathbf{q} \cdot \mathbf{d}_{\nu}} R_{0}\left(t^{\prime}\right) \\
& =\tau_{c} \sum_{\nu=1}^{N} R_{\mathrm{So}, \nu} \Gamma_{\nu} e^{-i \mathbf{q} \cdot \mathbf{d}_{\nu}} \int_{0}^{t} d t^{\prime} R_{0}\left(t-t^{\prime}\right) \frac{1}{\tau_{c}} R_{0}\left(t^{\prime}\right),
\end{aligned}
$$

with $R_{\mathrm{So}, v}$ being a function of $d_{\nu}$ which depends on the mechanism of the diffusive motion leading to the jumps from one site to another.

The Laplace transform of the series in Eq. (1) becomes

$$
\tilde{P}(s)=\frac{\tilde{R}_{0}(s)}{1-\tau_{c} \sum_{\nu=1}^{N} R_{\mathrm{so}, \nu} \Gamma_{\nu} e^{-i \mathbf{q} \cdot \mathbf{d}_{\nu}} \frac{1}{\tau_{c}} \tilde{R}_{0}(s)} .
$$

To evaluate the term $\sum_{\nu=1}^{N} R_{\mathrm{so}, \nu} \Gamma_{\nu} e^{-i \mathbf{q} \cdot \mathbf{d}_{\nu}}$, we first must know the form of $\sum_{v=1}^{N} \Gamma_{\nu} e^{-i \mathbf{q} \cdot \mathbf{d}_{\nu}}$. We know that $\tau_{c} \sum_{\nu=1}^{N} \Gamma_{\nu} e^{-i \mathbf{q} \cdot \mathbf{d}_{\nu}}=1-\tau_{c} \Gamma(q)$; in the CE model the sum $\sum_{v}$ is replaced by an integral over space and orientational averaging. It is possible to determine $\Gamma(q)$ whatever the jump length distribution from $\Gamma^{\mathrm{CE}}(q)$, the diffusion broadening obtained with the $\mathrm{CE}$ model, through the following relation: $\Gamma(q)=\int_{0}^{\infty} \Gamma^{\mathrm{CE}}(q) \rho(r) d r=\int_{0}^{\infty} \frac{1}{\tau_{c}}\left(1-\frac{\sin (q r)}{q r}\right) \rho(r) d r$, with $\rho(r)$ being the jump length distribution and for $\int_{0}^{\infty} \rho(r) d r=$ 1 . Then we get

$$
\tau_{c} \sum_{\nu=1}^{N} R_{\mathrm{so}, \nu} \Gamma_{\nu} e^{-i \mathbf{q} \cdot \mathbf{d}_{\nu}} \equiv \int_{0}^{\infty} \frac{\sin (q r)}{q r} R_{\mathrm{so}}(r) \rho(r) d r .
$$

When $q \rightarrow \infty$, the left and right terms in Eq. (27) tend to 0 , and $\tilde{P}(s)$ reduces to $\tau_{c} \tilde{R}_{0}(s)$ as expected. For small $q$, while $\sin (q r) \sim q r$, Eq. (27) gives $\bar{R}_{\mathrm{so}}$, the mean value of $R_{\mathrm{so}}$. For any $q, \int_{0}^{\infty} \frac{\sin (q r)}{q r} R_{\mathrm{so}}(r) \rho(r) d r=\hat{R}_{\mathrm{so}}(q) *\left[1-\tau_{c} \Gamma(q)\right]$, with * being the convolution product, which can be replaced by $\bar{R}_{\mathrm{so}}\left[1-\tau_{c} \Gamma(q)\right]$ when $R_{\mathrm{so}}(r)$ is a smooth function compared with the jump length distribution.

Let us discuss the case where each jump between localized sites consists of numerous fast (compared with $\tau_{c}$ ) and short-distance (compared with the characteristic jump length $l_{0}$ ) elementary jumps in random directions. One can assume, for example, fast hops between nearest neighbors separated by a distance $r_{c}$. At each hop the polarization decay due to spin-orbit coupling is given by $R_{\mathrm{so}}=1-\frac{1}{3} \gamma^{2}$, if the angular rotation $\gamma$ is small. Thus, when a spin at a site $\mathbf{r}_{\mathbf{i}}$ jumps to another site $\mathbf{r}_{\mathbf{i}}+\mathbf{d}_{v}$, it will experience $N_{v}=\left(d_{v} / r_{c}\right)^{2}$ uncorrelated rotations by an angle $\gamma$. Thus one can basically write $R_{\mathrm{So}, \nu}=1-\frac{1}{3} \gamma^{2}\left(\frac{d_{v}}{r_{c}}\right)^{2}$ or $R_{\mathrm{so}}(r)=1-\frac{1}{3} \gamma^{2}\left(\frac{r}{r_{c}}\right)^{2}$, and thus

$$
\bar{R}_{\mathrm{So}}=1-\frac{1}{3} \gamma^{2}\left(\frac{\bar{l}^{2}}{r_{c}^{2}}\right),
$$

where $\overline{l^{2}}=\int_{0}^{\infty} r^{2} \rho(r) d r$ is the mean-square displacement. If one introduces the spin-orbit length $L_{\mathrm{so}}$, which characterizes the jump distance at which the spin orientation is lost, we may

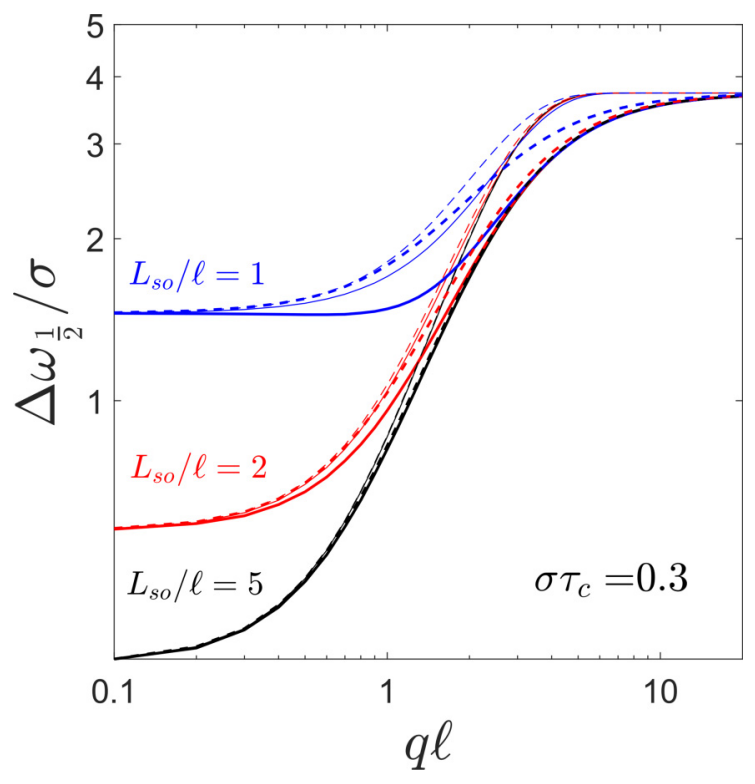

FIG. 2. Half-width at half maximum (HWHM) of the real part of Eq. (27) in reduced units for Gaussian (thin lines) and exponential (thick lines) jump length distribution, as a function of $q \ell$ with $\ell=$ $\sqrt{\bar{l}^{2}}$. Solid lines, Eq. (27) with $R_{\mathrm{so}}(r)=1-\frac{1}{3}\left(\frac{r}{L_{\mathrm{so}}}\right)^{2}$. Dashed lines, Eq. (27) assumed to be equal to $\bar{R}_{\mathrm{so}}\left[1-\tau_{c} \Gamma(q)\right]$.

write $\gamma=r_{c} / L_{\mathrm{so}}$ [57], so that, in fine,

$$
\bar{R}_{\mathrm{so}}=1-\frac{1}{3}\left(\frac{\overline{l^{2}}}{L_{\mathrm{so}}^{2}}\right)
$$

We note that this expression is identical to the one expected for a single jump between distant donors separated by an average mean-square distance $\overline{l^{2}}$.

Figure 2 shows the broadening of the line deduced from the real part of Eq. (26) calculated for Gaussian and exponential jump length distributions, for different values of the spin-orbit length and using Eq. (27) or its averaged form. For $L_{\mathrm{so}} \geqslant$ $5 \sqrt{\bar{l}^{2}}$ the relation $\bar{R}_{\mathrm{so}}\left[1-\tau_{c} \Gamma(q)\right]=\hat{R}_{\mathrm{so}}(q) *\left[1-\tau_{c} \Gamma(q)\right]$ is almost true, while for $L_{\mathrm{so}} \geqslant 2 \sqrt{\bar{l}^{2}}$ it is difficult in an experiment to differentiate between the convolution or the averaged forms.

Keeping the averaged form and using Eqs. (12) and (26), the $q$-resolved spin noise spectra $S_{0}(\omega, \mathbf{q})$ can be expressed as

$$
\begin{aligned}
S_{0}(\omega, \mathbf{q}) & =\frac{1}{\pi} \operatorname{Re}\left\{\frac{R(\omega)}{1-\bar{R}_{\mathrm{so}} F(\mathbf{q}) \frac{1}{\tau_{c}} R(\omega)}\right\}, \\
R(\omega) & =\frac{1}{\sigma} \sqrt{\frac{\pi}{2}} \mathcal{W}\left[\frac{\omega-\omega_{0}+i\left(v_{s}+1 / \tau_{c}\right)}{\sqrt{2} \sigma}\right], \\
F(\mathbf{q}) & =\frac{1}{N} \sum_{v=1}^{N} \exp \left(-i \mathbf{q} \cdot \mathbf{d}_{v}\right) .
\end{aligned}
$$

It contains the one-site response function $R(\omega)=\tilde{R}_{0}\left(s+v_{s}\right)$, and we have introduced the structure factor $F(\mathbf{q})=1-$ $\tau_{c} \Gamma(\mathbf{q})$. 


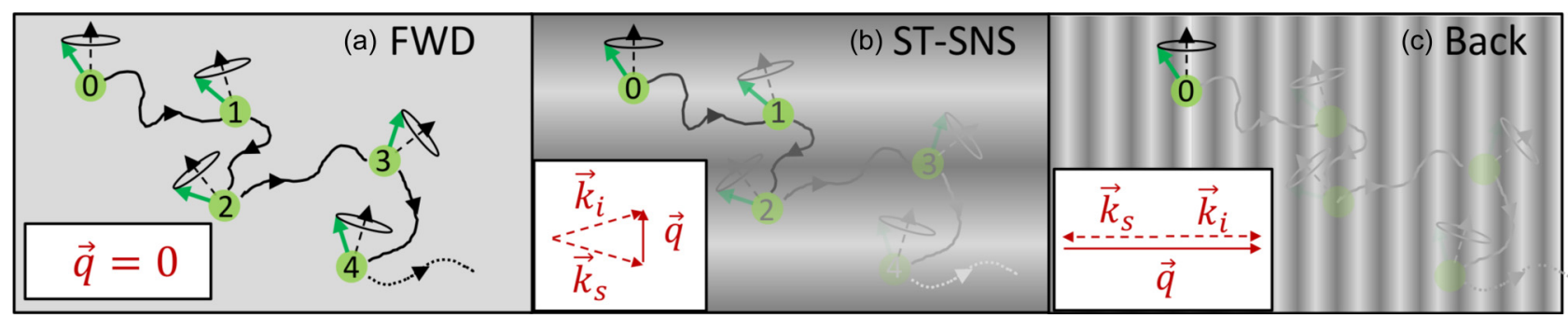

FIG. 3. Sketch of an electron spin trajectory along a chain of trapped states (green circles) starting from an initial state "0." Only the first five states are represented. We consider a spin initially in a bright fringe. The fringes represent the spin visibility, which depends on the relative phase between the incident (wave vector $\mathbf{k}_{i}$ ) and the scattered light (wave vector $\mathbf{k}_{s}$ ) (see Ref. [26]). The spin is represented as a green arrow and precesses in a local nuclear field (dashed arrow). The black curve represents the spin trajectory along fast diffusion channels. From left to right, three spin noise detection schemes used in this paper are represented. (a) Forward-scattering (FWD) geometry (classical SNS): Spin visibility is maximum and constant along the trajectory. All terms in Eq. (32) equally contribute to the signal. (b) ST-SNS: The spin visibility is slowly modulated. The terms contribute less and less as the number of jumps increases. (c) Backscattering (Back) geometry: When the modulation period of the spin visibility is smaller than the distance between traps, only the initial state "0" contributes to the signal (for random trap positions).

\section{Time of flight}

Next, we introduce the finite resolution in $q$ via the convolution [26]

$$
S(\omega, \mathbf{q})=\int_{-\infty}^{\infty} d^{2} \mathbf{q}^{\prime} S_{0}\left(\omega, \mathbf{q}^{\prime}\right) \hat{I}_{0}^{2}\left(\mathbf{q}-\mathbf{q}^{\prime}\right),
$$

where $\hat{I}_{0}(\mathbf{q})=e^{-q^{2} / 2 Q^{2}} /(\sqrt{\pi} Q)$ is the wave-vector distribution of the probe beam, with $Q=\sqrt{8 \ln (2)} / w$, where $w$ is the laser spot size on the sample. For a distribution that is not too large, one finds an analytical expression (see Appendix A)

$$
\begin{aligned}
S(\omega, \mathbf{q}) & =\frac{1}{\pi} \operatorname{Re}\left\{\frac{R(\omega)}{1-\bar{R}_{\mathrm{so}} G(\mathbf{q}) \frac{1}{\tau_{c}} R(\omega)}\right\}, \\
G(\mathbf{q}) & =F(\mathbf{q})+\frac{1}{4} Q^{2} F^{(2)}(\mathbf{q}) .
\end{aligned}
$$

$F^{(2)}(\mathbf{q})$ is the second derivative (Laplacian in $q$ space) of the structure factor $F(\mathbf{q})$. The corresponding term in Eq. (32) can be interpreted as the polarization decay due to time of flight (TOF) of the spin fluctuation across the probed area [58]. In effect the associated broadening is inversely proportional to the time for the spin fluctuation to leave the sampling area.

Equations (30) and (32) are only valid at large external field satisfying $\omega_{0} \gg \sigma$. To obtain the noise spectra in zero field, we use for $R(\omega)$ the one-site response calculated by Glazov \{the term $\tau_{\omega} A\left(\tau_{\omega}\right)$ in Eqs. (6)-(9) of Ref. [52] $\}$.

\section{Interpretation}

Equation (30) has a simple interpretation. $R(\omega)$ is the response of spins during their residence time on one site. At each step of the random walk, the spin coherence decays by a constant factor, which adds, to the decoherence during the time spent in the trap described by $\frac{1}{\tau_{c}} R(\omega)$, the spin rotation induced by SOC during the jump described by $1-\bar{R}_{\text {so }}$, and the structure factor $F(\mathbf{q})$. Hence Eq. (30) simply results from the summation of a geometric series, where the term of order $p$ corresponds to $p$ jumps.

Figure 3 graphically represents how the different terms of the geometric series contribute to the signal depending on the different detection schemes we use. Only one spin trajectory is represented, and we disregard TOF effects $(Q=0)$ and relaxation by $\operatorname{SOC}\left(\bar{R}_{\text {so }}=1\right)$. In backscattering, where $q$ is much larger than the inverse of the jump distance, the structure factor $F(q)$ averages to zero, and $S(\omega, q) \propto \operatorname{Re}[R(\omega)]$. The spectrum corresponds to the one-site response, in agreement with Fig. 3(c). Under an applied magnetic field, it follows an exact Voigt profile with $\sigma$ as Gaussian parameter and $\gamma=1 / \tau_{c}+v_{s}$ as Lorentzian parameter.

In classical SNS $[q=0$, Fig. 3(a)], $F(0)=1$, so that all terms contribute. In other words, one follows the spin all along the trajectory, and the signal results from averaging over an ensemble of such trajectories. $S(\omega, q)$ remains very close to a Voigt profile, but the Gaussian parameter is smaller than $\sigma$ : The linewidth decreases by motional narrowing of the local nuclear fields. In ST-SNS [Fig. 3(b)] a finite number of terms contribute; in other words, the trajectory fades away. This adds a broadening mechanism linked to motion, quadratic at small $q(q \ell \leqslant 1)$, from which the spin diffusion coefficient $D_{s}$ can be deduced. Finally, in the presence of SOC a competition sets in between broadening due to relaxation by SOC and motional narrowing.

\section{SPIN NOISE EXPERIMENTS}

We studied an Al-doped CdTe layer grown by molecular beam epitaxy on a $\mathrm{Cd}_{0.96} \mathrm{Zn}_{0.04} \mathrm{Te}(001)$ substrate, mechanically polished on its back face. The donor density, below the Mott transition, was estimated from secondary ion mass spectroscopy to be $\sim 2 \times 10^{16} \mathrm{~cm}^{-3}$, corresponding to an average interdonor distance of $\sim 50 \mathrm{~nm}$. The layer thickness is $10 \mu \mathrm{m}$. In a previous study we have observed a satellite line in the zero-field spin noise spectra of this sample (M3460), assigned to the electron spin precession in the local nuclear field $\sim 1.2 \mathrm{mT}$ [27]. A similar effect was also observed in high-purity GaAs [59].

Details of the experimental setup can be found in Refs. [26,27]. Here, the laser was detuned $9.3 \mathrm{meV}$ below the donor-bound exciton at $E=1.5926 \mathrm{eV}$ in a region of low absorption. Spin noise spectra with and without applied magnetic field were obtained simultaneously by repeated 

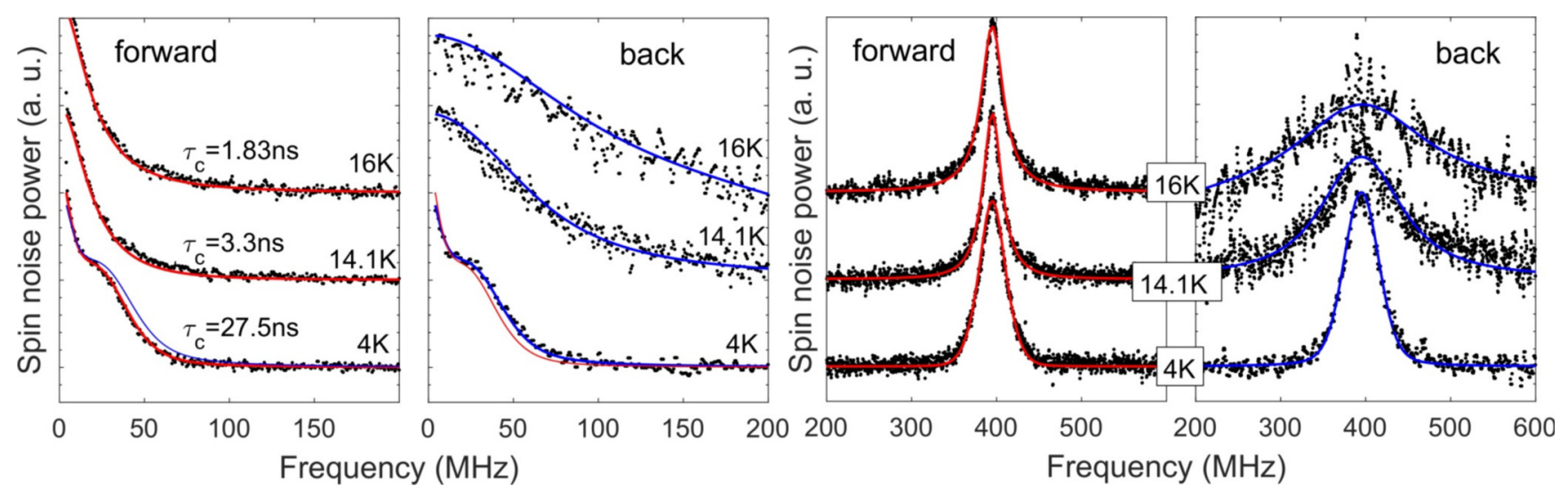

FIG. 4. Series of spin noise spectra for three different temperatures in forward scattering and backscattering (self-homodyne detection), in zero field (left) and in a transverse magnetic field (right). The curves are calculated with Eq. (32). Red (blue) curves are calculated at $q=0$ $(q=\infty)$. At $4 \mathrm{~K}$ and $B=0$ we highlight the difference between forward scattering and backscattering by plotting the curves calculated for both configurations (same color code).

interleaved acquisition in a single experimental run. The applied field $16 \mathrm{mT}$ was chosen much larger than the local nuclear field.

Figure 4 shows typical spin noise spectra, in forward scattering and backscattering (intermediate spectra using ST-SNS are not shown). A good fit was obtained for the spectra with field using Voigt profiles, for all values of $q$, and the halflinewidth at half maximum $\Delta \omega_{\frac{1}{2}}(q)$ is shown in Fig. 5(a) [60].

The strong dependence on $q$ is evidence of the role of spin motion. Figure 5(a) shows that $\Delta \omega_{\frac{1}{2}}(q)$ increases quadratically at small $q$ (see also Ref. [26]): This is the usual continuous diffusion regime described by a diffusion coefficient $D_{s}$ with a diffusion damping rate $\gamma_{\text {diff }}=D_{s} q^{2}$. However, $\Delta \omega_{\frac{1}{2}}(q)$ exhibits a plateau at large $q$. This behavior suggests that a characteristic length $\ell$ in the micrometer range characterizes the motion. Below we will interpret $\ell$ as the mean hopping distance and show that indeed the regime $q \ell \gg 1$ is reached for the backscattering spectra. Under such conditions [Fig. 3(c)], the signal is related to the one-site response: It can be characterized by an inhomogeneous broadening \{for instance, the Gaussian distribution of precession frequencies in the trapped states $p(\omega)=\left(2 \pi \sigma^{2}\right)^{-1 / 2} \exp \left[-\left(\omega-\omega_{0}\right)^{2} / 2 \sigma^{2}\right]$ due to hyperfine interaction (HFI) $\}$, an intrasite spin relaxation rate $v_{s}$ (ISR), and the spin residence time $\tau_{c}$ (hopping probability $1 / \tau_{c}$ ). The near-Gaussian profile at $4 \mathrm{~K}$ suggests that the linewidth is inhomogeneous and a good estimate of $\sigma$. The evolution towards a broader Lorentzian profile in backscattering $(q=\infty)$ as the temperature is increased shows that $v_{s}+1 / \tau_{c}$ increases. The evolution towards a narrower Lorentzian profile in forward scattering $(q=0)$ is interpreted as the result of motional narrowing, with an expected linewidth $\sim \sigma^{2} \tau_{c}$. Finally, the broad Lorentzian profile at higher temperature is interpreted as due to the increased role of time of flight (TOF; $\sim \frac{\ell^{2}}{w^{2} \tau_{c}}$ ) and spin-orbit coupling (SOC; $\sim \frac{\ell^{2}}{L_{\mathrm{so}}^{2} \tau_{c}}$ ): Both rates increase as $\tau_{c}$ decreases.

Those trends are schematized in Fig. 5(b).

A quantitative check using Eq. (32) is shown in Figs. 4 and 5. We obtain a simultaneous agreement between all experimental and theoretical spectra (including those at finite $q$ ) for a Gaussian distribution with $\ell=2.7 \mu \mathrm{m}$, and for $\sigma=1.12 \times 10^{8} \mathrm{~s}^{-1}$ and $L_{\mathrm{so}}=5 \mu \mathrm{m}$ (see Appendix $\mathrm{C}$ for details of the fitting procedure).

The value $w=16.8 \mu \mathrm{m}$ is estimated from the measurement of the spot size. The main parameter which changes with temperature is $\tau_{c}$. In order to reproduce the line shape of the satellite at $q=0$, we have to assume an additional broadening mechanism at $4 \mathrm{~K}$. Although the origin of this broadening is still unclear, we take it into account by assuming

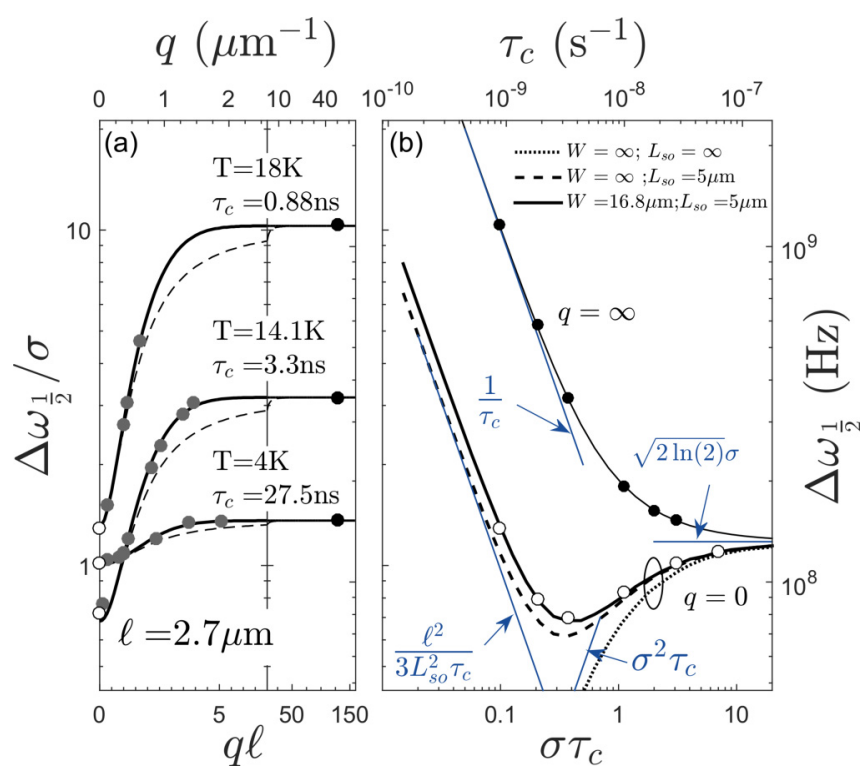

FIG. 5. HWHM $\Delta \omega_{\frac{1}{2}}$ vs $q$ (a) and vs $\tau_{c}$ (b). Experimental values (symbols) are measured at $16 \mathrm{mT}$ (probe laser power $P_{\ell}=2 \mathrm{~mW}$ ). Data are obtained by self-homodyne detection either in forward scattering (open circles) or backscattering (dark circles), and by ST-SNS (gray circles). All curves are calculated using Eq. (32) (parameters in the text). In (a), $\Delta \omega_{\frac{1}{2}}(q)$ is calculated for a Gaussian (solid lines) and an exponential distribution of jump lengths (dashed lines). In (b) the thin solid black line is calculated for $q=\infty$; the other black lines are calculated for $q=0$ with only HFI included (dotted line), $\mathrm{HFI}+\mathrm{SOC}$ (dashed line), HFI + SOC + TOF (thick solid line). The asymptotic behaviors are also indicated for $\sigma \tau_{c} \rightarrow 0$ and $\sigma \tau_{c} \rightarrow \infty$. The rightmost point in (b) was obtained at $P_{\ell}=0.25 \mathrm{~mW}$. 


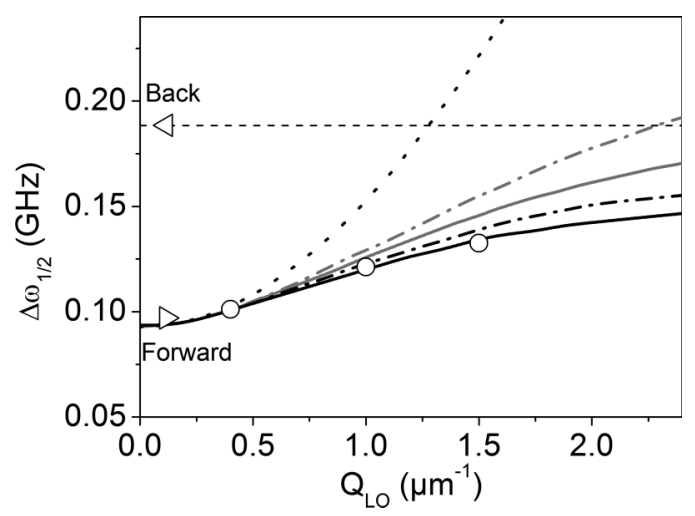

FIG. 6. HWHM $\Delta \omega_{1 / 2}$ of the spin noise line as a function of the wave-vector distribution $Q_{\mathrm{LO}}$ of the LO beam, experiments (symbols: triangles and circles, self-homodyne and homodyne detection, respectively) and models (lines). The black solid line shows the calculation with Eq. (31) including the optical transfer function of the collection long-working-distance objective (Mitutoyo $\times 10$ ), the dash-dotted black line shows the calculation with Eq. (31), and the dotted black line shows the calculation with Eq. (32). The calculations are done for a Gaussian jump length distribution, with $Q_{0}=$ $115000 \mathrm{~m}^{-1}, \tau_{c}=11 \mathrm{~ns}, \sigma=0.112 \mathrm{~ns}^{-1}, l=2.7 \mu \mathrm{m}, \bar{R}_{\mathrm{so}}=0.903$, and $q=0$ (except for the dashed black where $q=\infty$ ). Gray solid and dash-dotted lines show the calculation with Eq. (31) $(q=0)$ with and without the optical transfer function of the imaging system, respectively, and for Brownian motion with a diffusion coefficient $D_{s}=l^{2} / 6 \tau_{c}$.

a small ISR $\left[v_{s}=(2 \pi) \times 4.3 \mathrm{MHz}\right]$. Remarkably the satellite at zero field is better resolved in backscattering (see spectra at $4 \mathrm{~K}$ in Fig. 4). This difference is also well reproduced by our calculation. Indeed, in this situation only the initial state " 0 " not affected by spin motion is observed [Fig. 3(c)]. In other words, the one-site response is being probed. Finally, once $\tau_{c}$ and $\ell$ are determined, one deduces $D_{s}=\ell^{2} / 6 \tau_{c}$, which varies in the range $0.44-14 \mathrm{~cm}^{2} / \mathrm{s}$ from 4 to $18 \mathrm{~K}$.

The time-of-flight broadening appears in the expression of the spin noise spectrum in the same way as the spin-orbit coupling; it is then impossible to discriminate between these two mechanisms without knowing $D_{s}$ and the spatial extent of the beam. A way to differentiate between both mechanisms would be to measure the spin noise linewidth as a function of the spot size on the sample. However, focusing more or less may, for example, induce a temperature change and then a change in the correlation time. A better way is to use the homodyne detection technique in order to keep unchanged (and as large as possible) the spot size on the sample and to select, with a local oscillator (LO), the probed area in the detection plane. In this case, in the expression of $\hat{I}_{0}^{2}(\mathbf{q})$, $Q^{2}$ must be changed by $\frac{1}{2}\left(Q_{0}^{2}+Q_{\mathrm{LO}}^{2}\right)$, where $Q_{0}$ and $Q_{\mathrm{LO}}$ are linked to the widths of the wave-vector distributions of the beam probing the sample and the local oscillator, respectively. Calculations and measurements are depicted in Fig. 6. In the experiment a large probe beam $\left(Q_{0}=115000 \mathrm{~m}^{-1}\right)$ illuminates the sample, while a LO beam with a varying $Q_{\text {Lo }}$ probes the correlations in the sample's image. Triangles are for self-homodyne measurements (without the LO beam) in transmission (forward) and reflection (back), while open circles correspond to homodyne measurement (with LO beam) in transmission. Calculations are made taking into account or not the transfer function of the imaging system. When optical transfer function $h(\mathbf{q})$ is considered, $S(\omega, \mathbf{q})$ must be replaced by $S(\omega, \mathbf{q}) h(\mathbf{q})$; this case is never considered in the approximate solution given by Eq. (32).

We found that the broadening due to the TOF agrees well with the calculated one. This is important to deduce the value of $\Delta \omega_{\frac{1}{2}}(q)$ corrected for the TOF [Fig. 5(b)].

\section{CONCLUSION}

To conclude, our experiments have uncovered a spin-jumpdiffusion regime in an n-doped CdTe layer, which manifests as a plateau at large wave vectors for the broadening of the $q$-resolved spin noise spectra. We have proposed phenomenological equations, which simultaneously fit all spin noise spectra in zero and nonzero magnetic field, for all $q$ vectors and temperatures, with a fixed set of parameters $\sigma, L_{\mathrm{so}}$, and $\ell$. Besides, $\tau_{c}$ decreases with temperature and is mainly determined from the width of the noise spectra in backscattering. Remarkably, this determination of $\tau_{c}$ is consistent with the observation or not of the satellite line. It turns out that the parameters which govern the spin relaxation by HFI and SOC in CdTe, $\sigma$ and $L_{\mathrm{so}}$, respectively, are quite similar to the values in $\operatorname{GaAs}\left(\sigma=1.29 \times 10^{8} \mathrm{~s}^{-1}\right.$ [59], $L_{\mathrm{so}} \simeq 5 \mu \mathrm{m}$ [57] $)$. Systematic measurements for different doping levels will be necessary for a quantitative comparison with GaAs. However, we have strong indications that the faster relaxation in $\mathrm{CdTe}$ is not due to material parameters, but rather to the specific long-distance spin jump diffusion that we have uncovered. The inhomogeneities of the system may plausibly play an important role in this phenomenon [61-63].

Our experimental value of $L_{\mathrm{so}}$ is close to the theoretical value $L_{\mathrm{so}} \simeq 3.37 E_{\mathrm{b}} a_{\mathrm{b}}^{4} / \gamma_{\mathrm{c}}=4.1 \mu \mathrm{m}$ [57], where $E_{\mathrm{b}}=$ $13 \mathrm{meV}$ is the donor binding energy, $a_{\mathrm{b}}=5.3 \mathrm{~nm}$ is its Bohr radius, and $\gamma_{\mathrm{c}}=8.5 \mathrm{eV} \AA^{3}$ is the Dresselhaus coefficient taken from theoretical estimations [64]. $\sigma$ can be estimated from the Bohr radius and the hyperfine constants of $\mathrm{Cd}$ and Te. Unfortunately, reliable values for the hyperfine constants are missing (see discussion in Ref. [27]). The longest spin relaxation time, corrected for a small TOF effect, that we measure in the regime of motional narrowing is $T_{2}^{*}=15 \mathrm{~ns}$ (see Appendix B for the precise definition of $T_{2}^{*}$ and how it is determined).

The long-range spin hopping uncovered here in CdTe has important consequences for the electron spatiotemporal spin dynamics and hence for qubit operations based on donors: It should be sought in other materials which are under focus for quantum technologies [3,45,47], such as GaAs, $\mathrm{ZnSe}, \mathrm{ZnO}$, and, if possible, Si and Ge. Our approach could also answer fundamental questions which underpin such operations, among which is the predicted random spin rotation during a hop between two donors, which arises due to the uncertainty in the quantum trajectory [61].

\section{ACKNOWLEDGMENTS}

S.C. and D.S. wish to thank C. Lhenoret for technical assistance. 


\section{APPENDIX A: TIME OF FLIGHT}

The spin may diffuse outside of the probed area leading to an additional broadening [58] that we will call time-of-flight broadening because it is inversely proportional to the time for the spin to leave the sampling area. It can be taken into account by a convolution of the $q$-dependent spectrum with the wave-vector distribution of the probe beam intensity [26],

$$
S(\omega, \mathbf{q})=\int_{-\infty}^{\infty} d^{2} \mathbf{q}^{\prime} S\left(\omega, \mathbf{q}^{\prime}\right) \hat{I}_{0}^{2}\left(\mathbf{q}-\mathbf{q}^{\prime}\right)=\frac{1}{\pi} \int_{-\infty}^{\infty} d^{2} \mathbf{q}^{\prime} \operatorname{Re}\left\{\frac{R(\omega)}{1-\bar{R}_{\mathrm{so}} F\left(\mathbf{q}^{\prime}\right) \frac{1}{\tau_{c}} R(\omega)}\right\} \hat{I}_{0}^{2}\left(\mathbf{q}-\mathbf{q}^{\prime}\right),
$$

with $\hat{I}_{0}(\mathbf{q})$ being the space Fourier transform of $I_{0}(\mathbf{r})$, the intensity profile of the probe beam. It is possible to give a simple analytical expression of integral in Eq. (A1) by using the Taylor series expansion of $F\left(\mathbf{q}^{\prime}\right)$ at the measured $\mathbf{q}$,

$$
F\left(\mathbf{q}^{\prime}\right)=F(\mathbf{q})+\left[\nabla_{q^{\prime \prime}} F(\mathbf{q})\right] \cdot\left(\mathbf{q}^{\prime}-\mathbf{q}\right)+\frac{1}{2} \nabla_{q^{\prime \prime}}\left\{\left[\nabla_{q^{\prime \prime}} F(\mathbf{q})\right] \cdot\left(\mathbf{q}^{\prime}-\mathbf{q}\right)\right\} \cdot\left(\mathbf{q}^{\prime}-\mathbf{q}\right)
$$

with $\nabla_{q^{\prime \prime}} F(\mathbf{q})=\left[\frac{\partial F\left(\mathbf{q}^{\prime \prime}\right)}{\partial q_{x}^{\prime \prime}}\right]_{\mathbf{q}^{\prime \prime}=\mathbf{q}} \mathbf{e}_{\mathbf{x}}+\left[\frac{\partial F\left(\mathbf{q}^{\prime \prime}\right)}{\partial q_{y}^{\prime \prime}}\right]_{\mathbf{q}^{\prime \prime}=\mathbf{q}} \mathbf{e}_{\mathbf{y}}$. Then, if $\hat{I}_{0}(\mathbf{q})$ is symmetrical, the convolution integral (A1) reduces to an integral,

$$
S(\omega, \mathbf{q})=\frac{1}{\pi} \int_{-\infty}^{\infty} d^{2} \mathbf{q}^{\prime} \operatorname{Re}\left\{\frac{R(\omega)}{1-\bar{R}_{\mathrm{so}}\left(F(\mathbf{q})+\left[\nabla_{q^{\prime \prime}} F(\mathbf{q})\right] \cdot \mathbf{q}^{\prime}+\frac{1}{2} \nabla_{q^{\prime \prime}}\left\{\left[\nabla_{q^{\prime \prime}} F(\mathbf{q})\right] \cdot \mathbf{q}^{\prime}\right\} \cdot \mathbf{q}^{\prime}\right) \frac{1}{\tau_{c}} R(\omega)}\right\} \hat{I}_{0}^{2}\left(\mathbf{q}^{\prime}\right) .
$$

If the wave-vector distribution of the beam is not too large or if $F(q)$ varies a little in the width of this distribution such that $\left[\nabla_{q^{\prime \prime}} F(\mathbf{q})\right] \cdot \mathbf{q}^{\prime}+\frac{1}{2} \nabla_{q^{\prime \prime}}\left\{\left[\nabla_{q^{\prime \prime}} F(\mathbf{q})\right] \cdot \mathbf{q}^{\prime}\right\} \cdot \mathbf{q}^{\prime} \ll F(\mathbf{q})$, the integration can be put directly in the denominator,

$$
S(\omega, \mathbf{q})=\frac{1}{\pi} \operatorname{Re}\left\{\frac{R(\omega)}{1-\bar{R}_{\mathrm{so}}\left[F(\mathbf{q})+\int_{-\infty}^{\infty} d^{2} \mathbf{q}^{\prime} \hat{I}_{0}^{2}\left(\mathbf{q}^{\prime}\right)\left(\left[\nabla_{q^{\prime \prime}} F(\mathbf{q})\right] \cdot \mathbf{q}^{\prime}+\frac{1}{2} \nabla_{q^{\prime \prime}}\left\{\left[\nabla_{q^{\prime \prime}} F(\mathbf{q})\right] \cdot \mathbf{q}^{\prime}\right\} \cdot \mathbf{q}^{\prime}\right)\right] \frac{1}{\tau_{c}} R(\omega)}\right\} .
$$

Linear in $q^{\prime}$ and mixed second-order partial derivative terms are equal to 0 after integration. For a Gaussian beam of the form $\hat{I}_{0}^{2}(\mathbf{q})=e^{-q^{2} / Q^{2}} /\left(\pi Q^{2}\right)$ the integral reduces to $\frac{1}{4} Q^{2} F^{(2)}(\mathbf{q})$, and $S(\omega, \mathbf{q})$ becomes

$$
S(\omega, \mathbf{q})=\frac{1}{\pi} \operatorname{Re}\left\{\frac{R(\omega)}{1-\bar{R}_{\mathrm{so}}\left[F(\mathbf{q})+\frac{1}{4} Q^{2} F^{(2)}(\mathbf{q})\right] \frac{1}{\tau_{c}} R(\omega)}\right\},
$$

with $F^{(2)}(\mathbf{q})=\left[\frac{\partial^{2} F\left(\mathbf{q}^{\prime}\right)}{\partial q_{x}^{\prime 2}}\right]_{\mathbf{q}^{\prime}=\mathbf{q}}+\left[\frac{\partial^{2} F\left(\mathbf{q}^{\prime}\right)}{\partial q_{y}^{\prime 2}}\right]_{\mathbf{q}^{\prime}=\mathbf{q}}$.

$\gamma_{\mathrm{TOF}}=-\frac{Q^{2} F^{(2)}(\mathbf{q})}{4 \tau}$ is then the broadening due to the probability of the spin leaving the sampling area. In a transmission experiment with $q=0, F(0)=1$, and $\gamma_{\mathrm{TOF}}=\frac{Q^{2} \vec{l}^{2}}{6 \tau_{c}}=Q^{2} D_{s}$, with $D_{s}$ being the spin diffusion coefficient, whatever the jump length distribution.

\section{APPENDIX B: LINE SHAPE, LINEWIDTH, AND SPIN RELAXATION TIME}

Let us consider the spin noise line whose spectrum at any wave vector, in the presence of hyperfine interaction, spinorbit coupling, and time of flight, is given by Eq. (A5). Under an external magnetic field applied transverse to the incident light, $R(\omega)$ identifies with $\tilde{R}_{0}\left(s+v_{s}\right), \tilde{R}_{0}(s)$ being given by Eq. (8). For the two limiting cases $\sigma \tau_{c} \gg 1$ and $\sigma \tau_{c} \ll 1$, one can easily deduce the line shape, which will be Gaussian in the former case and Lorentzian in the latter case. The damping parameter $\Lambda$, defined as the inverse of the time during which the temporal signal has decayed by $1 / e$, is a known parameter for both cases. $\Lambda$ equals the damping rate $\gamma$ for a Lorentzianshaped line and the square root of half the variance for a Gaussian-shaped line. Thus having acquaintance with the line shape, knowing the linewidth, one can deduce the damping parameter. This is true only for short and long $\tau_{c}$. Indeed, there is no analytical expression for the damping parameter corresponding to Eq. (A5) for any $\sigma \tau_{c}$, neither for the width of the line, nor for the relation between these two parameters. It is then difficult to estimate correctly the spin relaxation time from the noise power spectrum. A good approximation of the spin relaxation time is half the amplitude of the spin noise line when its surface is normalized to unity. This relaxation time, called $T_{2}^{*}$ and introduced by Bloembergen et al. [65], is defined by

$$
T_{2}^{*}=\frac{\frac{1}{2} g(\nu)_{\max }}{\int d \nu g(\nu)}=\frac{\pi g(\omega)_{\max }}{\int d \omega g(\omega)}
$$

where $g(v)[g(\omega)]$ is the spectrum in the frequency (pulsation) $v(\omega)$ space and $g(v)_{\max }\left[g(\omega)_{\max }\right]$ is its maximum amplitude. $\left(T_{2}^{*}\right)^{-1}$ equals the damping rate for a Lorentzian-shaped line and overestimates by an amount of less than $13 \%$ the damping parameter for a Gaussian-shaped line, so that $\left(T_{2}^{*}\right)^{-1} \approx \Lambda$ and one can call it the damping parameter. $T_{2}^{*}$ is thus a good estimate of the spin relaxation time; moreover, it can be estimated experimentally without any assumption as to the line shape. A joint determination of $\left(T_{2}^{*}\right)^{-1}$ and the half-width at half maximum $\Delta \omega_{1 / 2}$ gives information about the line shape. If $\left(T_{2}^{*}\right)^{-1} / \Delta \omega_{1 / 2}=1$, the line is Lorentzian, and if $\left(T_{2}^{*}\right)^{-1} / \Delta \omega_{1 / 2}=0.678$, the line is Gaussian.

Definition (B1) applied to Eq. (A5) leads to the following expression for the damping parameter:

$$
\left(T_{2, q}^{*}\right)^{-1}=R^{-1}\left(\omega_{0}\right)-\frac{\eta(q)}{\tau_{c}},
$$




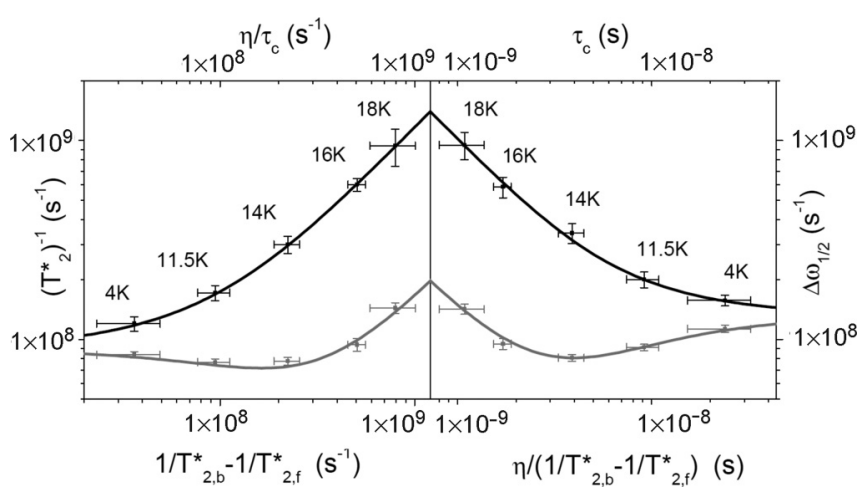

FIG. 7. Left: $\left(T_{2, b / f}^{*}\right)^{-1}$ vs $\left(T_{2, b}^{*}\right)^{-1}-\left(T_{2, f}^{*}\right)^{-1}$ deduced with Eq. (B1) from the measured spin noise power spectrum for two different detection schemes: backscattering SNS $\left[\left(T_{2, b}^{*}\right)^{-1}\right]$ and classical SNS $\left[\left(T_{2, f}^{*}\right)^{-1}\right]$ (black and gray points with error bars, respectively). $\left(T_{2}^{*}\right)^{-1}$ deduced from Eqs. (A5) and (B2) for $q=0$ (gray solid line) and $q=\infty$ (black solid line). Right: Half-width at half maximum $\Delta \omega_{1 / 2}$ from experiments (points with error bars) and from Eq. (A5) (solid lines) [black and gray stand for backscattering SNS $(q=\infty)$ and classical SNS ( $q=0)$, respectively].

with $\eta(q)=\bar{R}_{\mathrm{so}} G(\mathbf{q})$. Let us discuss this quite interesting formulation for the case $q=0$ and $\eta(0)=1$. In this case, Eq. (B2) expresses the line broadening due to hyperfine interaction only, leading to motional narrowing with decreasing $\tau_{c}$. $R_{0}^{-1}\left(\omega_{0}\right)$ is the broadening of a Voigt profile which mixes the inhomogeneous broadening due to hyperfine interaction and the homogeneous broadening due to the probability $1 / \tau_{c}$ that the spin leaves a localized site. This homogeneous broadening does not contribute in a classical measurement $(q=0)$; it is then subtracted by the second term in Eq. (B2), so that only the competition between the inhomogeneous and the homogeneous parts remains. At short $\tau_{c}$ this is quite visible because the line broadening is proportional to $\sigma \frac{\sigma}{\gamma}$, with $\gamma=1 / \tau_{c}$ being the damping rate of the homogeneous part. When $\eta<1$, the other sources of broadening add simply as homogeneous terms.

When looking at the damping parameter in the two limiting cases $q=0$ and $q=\infty$, they differ only by the quantity $\eta(0) / \tau_{c}$. Then a measure of the difference $\left(T_{2, \infty}^{*}\right)^{-1}-\left(T_{2,0}^{*}\right)^{-1}$ is a direct determination of $\eta(0) / \tau_{c}$. Plotting $\left(T_{2, q}^{*}\right)^{-1}$ as a function of $\left(T_{2, \infty}^{*}\right)^{-1}-\left(T_{2,0}^{*}\right)^{-1}$ will place the data automatically at the right abscissa position getting rid of one fitting parameter, the correlation time $\tau_{c}$.

In Fig. 7 we plot $\left(T_{2}^{*}\right)^{-1}$ and $\Delta \omega_{1 / 2}$ extracted manually (without using any fitting function) from the noise power spectrum in measurements made on the n-CdTe sample described in the main text, under a transverse magnetic field of $16 \mathrm{mT}$, for different temperatures and for two different detection schemes [backscattering SNS, $\left(T_{2, b}^{*}\right)^{-1}$ from noise of the reflected beam; forward scattering SNS, $\left(T_{2, f}^{*}\right)^{-1}$ from noise of the transmitted beam]. On the left-hand side, the experimentally determined $\left(T_{2, b / f}^{*}\right)^{-1}$ are plotted as a function of their differences, while $\left(T_{2, q}^{*}\right)^{-1}$ calculated with Eq. (B2) for $q=0$ and $q=\infty$ is plotted as a function of $\eta / \tau_{c}$, with $\eta=\eta(q=0)$. A good agreement between theory and experiment is obtained for $\sigma=1.12 \times 10^{8} \mathrm{~s}^{-1}, \eta=0.87$, and

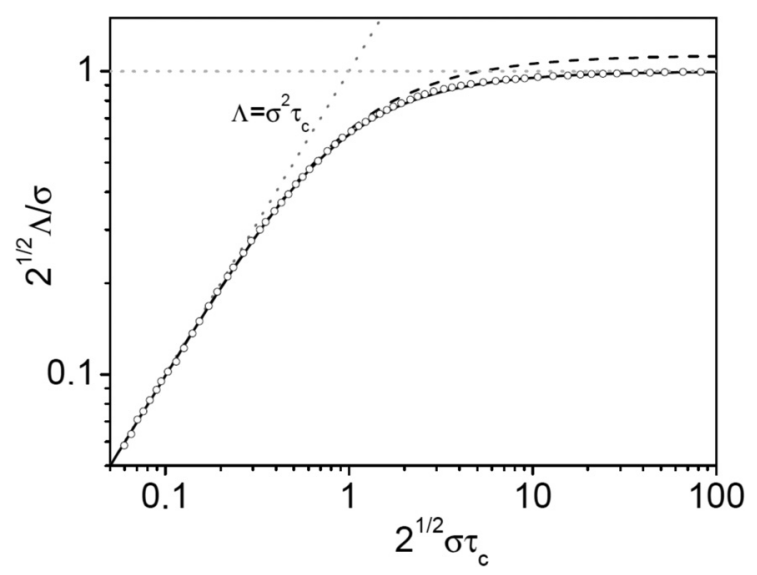

FIG. 8. Comparison of the damping constant $\Lambda / \sigma$ for the Markovian random-walk model (symbols; extracted from Fig. 3 of Ref. [50]), Eq. (B2) (dashed black line), and Eq. (B3) (solid black line).

$v_{s}=0$. On the right-hand side, the experimentally determined half-width at half maximum is plotted as a function of $\eta /\left[\left(T_{2, b}^{*}\right)^{-1}-\left(T_{2, f}^{*}\right)^{-1}\right]$, while the numerical solution of the equation $S\left(\Delta \omega_{1 / 2}, q\right)=S\left(\omega_{0}, q\right) / 2$ (with $\sigma, \eta$, and $v_{s}$ determined previously) for $q=0$ and $q=\infty$ is plotted as a function of $\tau_{c}$. From the ratio $\left(T_{2}^{*}\right)^{-1} / \Delta \omega_{1 / 2}$, one can deduce that the line is Lorentzian at short $\tau_{c}$ (high temperature $T>16$ $\mathrm{K}$ ) and almost Gaussian at long $\tau_{c}$ (low temperature $T=4 \mathrm{~K}$ ).

Finally, inspired by Eq. (B2), one can attempt to give an analytical expression for the damping parameter $\Lambda$ of Eq. (5) using the damping parameter of $R_{0}(t)=$ $\exp \left(-\frac{1}{2} \sigma^{2} t^{2}\right) \exp \left(-t / \tau_{c}\right)$. Let us call $\Lambda_{\sigma, \tau_{c}}$ the damping parameter of $R_{0}(t)$, such that $R_{0}\left(\Lambda_{\sigma, \tau_{c}}^{-1}\right)=1 / e$. Following the structure of Eq. (B2), we propose

$$
\Lambda=\Lambda_{\sigma, 2 \tau_{c}}-\frac{1}{2 \tau_{c}} .
$$

In Fig. 8 we compare Eq. (B2) [for $\eta(q=0)=1$ ] and Eq. (B3) with $\Lambda$ extracted from Fig. 3 of Ref. [50], deduced from $P(t)$ obtained by inversion of the Laplace transform of Eq. (6). We find a maximum deviation of $1.7 \%$ for $\sigma \tau_{c} \approx \sqrt{\pi}$ between Eq. (B3) and $\Lambda$ extracted from Fig. 3 of Ref. [50].

When considering other sources of homogeneous broadening, then $\eta(q=0)<1$, so that Eq. (B3) is written as

$$
\Lambda=\Lambda_{\sigma, 2 \tau_{c}}-\frac{2 \eta(q)-1}{2 \tau_{c}}
$$

\section{APPENDIX C: DETERMINATION OF THE SPIN RELAXATION PARAMETERS}

In order to determine the spin relaxation parameters $\sigma, L_{\mathrm{so}}$, $\tau_{c}$, and $\ell$, we make use of the results presented in Appendixes $\mathrm{A}$ and $\mathrm{B}$, together with the $q$ dependence of $\Delta \omega_{\frac{1}{2}}$ [Fig. 5(a)].

As we have seen in Appendix B, using the spin relaxation rates is very convenient for this task because it allows for the direct determination of the parameter $\eta(q) / \tau_{c}$. However, the experimental determination of $\left(T_{2, q}^{*}\right)^{-1}$ requires a high enough signal-to-noise ratio in order to have a good estimate of the wings of the line, and of the background level. This is a bit 
more difficult to estimate in the ST-SNS configuration, where the optical transfer function of the collection objective filters out the signal as $q$ increases. Therefore we could determine precisely only $\left(T_{2, f}^{*}\right)^{-1}$ and $\left(T_{2, b}^{*}\right)^{-1}$ (see Fig. 7). This is why in Fig. 5 we plot only $\Delta \omega_{\frac{1}{2}}$. However, from Fig. 5 one can see that $T_{2, b}^{*} \equiv T_{2, \infty}^{*}$, while $T_{2, f}^{*} \equiv T_{2,0}^{*}$ is always true. Hence with Eq. (B2) we can write

$$
\begin{aligned}
T_{2, b}^{-1} & =R^{-1}\left(\omega_{0}\right), \\
T_{2, f}^{-1} & =T_{2, b}^{-1}-\frac{\eta}{\tau_{c}} .
\end{aligned}
$$

The difference between back and forward spin relaxation rates already fixes the value of the parameter $\eta / \tau_{c}$, where
$\eta=\bar{R}_{\mathrm{so}}\left[1-(Q \ell)^{2} / 6\right]$. At long $\tau_{c}$ (low temperatures) these two relaxation rates converge towards the common value $\sqrt{\frac{2}{\pi}} \sigma$. This approximately determines $\sigma$. At short $\tau_{c}$ (high temperatures), $\left(T_{2, b}^{*}\right)^{-1}$ tends towards $1 / \tau_{c}$. Thus one can roughly deduce both $\eta$ and $\tau_{c}$. Then the fine-tuning of the parameters is made by seeking for the best agreement for all temperatures between experiment and theory (see Fig. 7). We finally obtain $\sigma=1.12 \times 10^{8} \mathrm{~s}^{-1}$ and $\eta=0.87 . \tau_{c}$ is thus automatically determined at all temperatures.

We are left with the determination of $L_{\mathrm{so}}$ and $\ell . \ell=2.7 \mu \mathrm{m}$ is deduced from the variation of $\Delta \omega_{\frac{1}{2}}$ with $q$ [Fig. 5(a)]. Then from the measured width of the spot size $w=16.8 \mu \mathrm{m}$, we get $Q=140200 \mathrm{~m}^{-1}$; hence $\bar{R}_{\mathrm{so}}=0.903$, and finally $L_{\mathrm{so}}=$ $5 \mu \mathrm{m}$.
[1] C. D. Hill, L. C. L. Hollenberg, A. G. Fowler, C. J. Wellard, A. D. Greentree, and H.-S. Goan, Phys. Rev. B 72, 045350 (2005).

[2] J. J. Pla, K. Y. Tan, J. P. Dehollain, W. H. Lim, J. J. L. Morton, D. N. Jamieson, A. S. Dzurak, and A. Morello, Nature (London) 489, 541 (2012).

[3] D. D. Awschalom, L. C. Bassett, A. S. Dzurak, E. L. Hu, and J. R. Petta, Science 339, 1174 (2013).

[4] J. J. Morton, D. R. McCamey, M. A. Eriksson, and S. A. Lyon, Nature (London) 479, 345 (2011).

[5] A. Morello, J. J. Pla, P. Bertet, and D. N. Jamieson, Adv. Quantum Technol. 3, 2000005 (2020).

[6] A. D. Greentree, J. H. Cole, A. R. Hamilton, and L. C. L. Hollenberg, Phys. Rev. B 70, 235317 (2004).

[7] L. C. L. Hollenberg, A. D. Greentree, A. G. Fowler, and C. J. Wellard, Phys. Rev. B 74, 045311 (2006).

[8] R. Sánchez, G. Granger, L. Gaudreau, A. Kam, M. PioroLadrière, S. A. Studenikin, P. Zawadzki, A. S. Sachrajda, and G. Platero, Phys. Rev. Lett. 112, 176803 (2014).

[9] T. Fujita, T. A. Baart, C. Reichl, W. Wegscheider, and L. M. K. Vandersypen, npj Quantum Inf. 3, 22 (2017).

[10] H. Flentje, P.-A. Mortemousque, R. Thalineau, A. Ludwig, A. D. Wieck, C. Bäuerle, and T. Meunier, Nat. Commun. 8, 501 (2017).

[11] E. B. Aleksandrov and V. S. Zapasskii, Zh. Eksp. Teor. Fiz. 81, 132 (1981) [Sov. Phys. JETP 54, 64 (1981)].

[12] T. Mitsui, Phys. Rev. Lett. 84, 5292 (2000).

[13] S. A. Crooker, D. G. Rickel, A. V. Balatsky, and D. L. Smith, Nature (London) 431, 49 (2004).

[14] J. L. Sørensen, J. Hald, and E. S. Polzik, Phys. Rev. Lett. 80, 3487 (1998).

[15] A. Kuzmich, L. Mandel, and N. P. Bigelow, Phys. Rev. Lett. 85, 1594 (2000).

[16] K. Eckert, Ł. Zawitkowski, A. Sanpera, M. Lewenstein, and E. S. Polzik, Phys. Rev. Lett. 98, 100404 (2007).

[17] M. Oestreich, M. Römer, R. J. Haug, and D. Hägele, Phys. Rev. Lett. 95, 216603 (2005).

[18] J. Hubner, F. Berski, R. Dahbashi, and M. Oestreich, Phys. Status Solidi B 251, 1824 (2014).

[19] S. Cronenberger and D. Scalbert, Rev. Sci. Instrum. 87, 093111 (2016).
[20] M. Y. Petrov, A. N. Kamenskii, V. S. Zapasskii, M. Bayer, and A. Greilich, Phys. Rev. B 97, 125202 (2018).

[21] P. Sterin, J. Wiegand, J. Hübner, and M. Oestreich, Phys. Rev. Appl. 9, 034003 (2018).

[22] Y. V. Pershin, V. A. Slipko, D. Roy, and N. A. Sinitsyn, Appl. Phys. Lett. 102, 202405 (2013).

[23] G. G. Kozlov, I. I. Ryzhov, and V. S. Zapasskii, Phys. Rev. A 97, 013848 (2018).

[24] A. V. Poshakinskiy and S. A. Tarasenko, Phys. Rev. B 92 , 045308 (2015).

[25] G. M. Bruun, B. M. Andersen, E. Demler, and A. S. Sørensen, Phys. Rev. Lett. 102, 030401 (2009).

[26] S. Cronenberger, C. Abbas, D. Scalbert, and H. Boukari, Phys. Rev. Lett. 123, 017401 (2019).

[27] S. Cronenberger, C. Abbas, D. Scalbert, and H. Boukari, arXiv:1910.11805.

[28] A. R. Cameron, P. Riblet, and A. Miller, Phys. Rev. Lett. 76, 4793 (1996).

[29] S. G. Carter, Z. Chen, and S. T. Cundiff, Phys. Rev. Lett. 97, 136602 (2006).

[30] C. P. Weber, J. Orenstein, B. A. Bernevig, S.-C. Zhang, J. Stephens, and D. D. Awshalom, Phys. Rev. Lett. 98, 076604 (2007).

[31] J. D. Koralek, C. P. Weber, J. Orenstein, B. A. Bernevig, S.-C. Zhang, S. Mack, and D. D. Awschalom, Nature (London) 458, 610 (2009).

[32] L. Yang, J. D. Koralek, J. Orenstein, D. R. Tibbetts, J. L. Reno, and M. P. Lilly, Nat. Phys. 8, 153 (2011).

[33] L. Yang, J. D. Koralek, J. Orenstein, D. R. Tibbetts, J. L. Reno, and M. P. Lilly, Phys. Rev. Lett. 109, 246603 (2012).

[34] S. Chandrasekhar, Rev. Mod. Phys. 15, 1 (1943).

[35] Diffusion in Condensed Matter, edited by P. Heitjans and J. Karger (Springer, Berlin, 2005).

[36] F. Brauer and C. Castillo-Chavez, Mathematical Models in Population Biology and Epidemiology, Texts in Applied Mathematics, Vol. 40 (Springer, New York, 2012).

[37] T. Fujiwara, K. Ritchie, H. Murakoshi, K. Jacobson, and A. Kusumi, J. Cell Biol. 157, 1071 (2002).

[38] V. Mwaffo, R. P. Anderson, S. Butail, and M. Porfiri, J. R. Soc., Interface 12, 20140884 (2015).

[39] S. Kou, Manage. Sci. 48, 1086 (2002). 
[40] R. Cont and P. Tankov, Financial Modelling with Jump Processes, Chapman \& Hall/CRC Financial Mathematics Series (CRC, London, 2004).

[41] C. T. Chudley and R. J. Elliott, Proc. Phys. Soc., London 77, 353 (1961).

[42] K. S. Singwi and A. Sjölander, Phys. Rev. 119, 863 (1960).

[43] D. Richter and T. Springer, Phys. Rev. B 18, 126 (1978).

[44] I. A. Zaliznyak and S.-H. Lee, in Modern Techniques for Characterizing Magnetic Materials, edited by Y. Zhu (Springer, Boston, 2005), pp. 3-64.

[45] A. Greilich, A. Pawlis, F. Liu, O. A. Yugov, D. R. Yakovlev, K. Lischka, Y. Yamamoto, and M. Bayer, Phys. Rev. B 85, 121303(R) (2012).

[46] E. A. Zhukov, D. R. Yakovlev, A. Schwan, O. A. Yugov, A. Waag, L. W. Molenkamp, and M. Bayer, Phys. Status Solidi B 251, 1872 (2014).

[47] X. Linpeng, M. L. K. Viitaniemi, A. Vishnuradhan, Y. Kozuka, C. Johnson, M. Kawasaki, and K.-M. C. Fu, Phys. Rev. Appl. 10, 064061 (2018).

[48] K. M. Whitaker, S. T. Ochsenbein, A. L. Smith, D. C. Echodu, B. H. Robinson, and D. R. Gamelin, J. Phys. Chem. C 114, 14467 (2010).

[49] P. L. Hall and D. K. Ross, Mol. Phys. 36, 1549 (1978).

[50] K. W. Kehr, D. Richter, and G. Honig, Hyperfine Interact. 6, 219 (1979).

[51] N. J. Harmon and M. E. Flatté, Phys. Rev. Lett. 110, 176602 (2013).
[52] M. M. Glazov, Phys. Rev. B 91, 195301 (2015).

[53] P. L. Hall and D. K. Ross, Mol. Phys. 42, 673 (1981).

[54] H. C. Torrey, Phys. Rev. 92, 962 (1953).

[55] P. A. Bobbert, W. Wagemans, F. W. A. van Oost, B. Koopmans, and M. Wohlgenannt, Phys. Rev. Lett. 102, 156604 (2009).

[56] R. C. Roundy and M. E. Raikh, Phys. Rev. B 90, 201203 (2014).

[57] K. V. Kavokin, Semicond. Sci. Technol. 23, 114009 (2008).

[58] G. M. Müller, M. Römer, D. Schuh, W. Wegscheider, J. Hübner, and M. Oestreich, Phys. Rev. Lett. 101, 206601 (2008).

[59] F. Berski, J. Hübner, M. Oestreich, A. Ludwig, A. D. Wieck, and M. Glazov, Phys. Rev. Lett. 115, 176601 (2015).

[60] $\Delta \omega_{\frac{1}{2}}$ equals the damping rate for a Lorentzian line and is in excess by $66 \%$ for a Gaussian. A better estimation is given by $\left(T_{2}^{*}\right)^{-1}$ as defined in Ref. [65], in excess by only $12 \%$ for a Gaussian. However, its determination is experimentally more demanding (see Appendix B).

[61] I. S. Lyubinskiy, A. P. Dmitriev, and V. Y. Kachorovskii, JETP Lett. 85, 55 (2007).

[62] I. S. Lyubinskiy, JETP Lett. 88, 814 (2008).

[63] G. Garcia-Arellano, F. Bernardot, G. Karczewski, C. Testelin, and M. Chamarro, Phys. Rev. B 102, 165314 (2020).

[64] A. N. Chantis, M. van Schilfgaarde, and T. Kotani, Phys. Rev. Lett. 96, 086405 (2006).

[65] N. Bloembergen, E. M. Purcell, and R. V. Pound, Phys. Rev. 73, 679 (1948). 